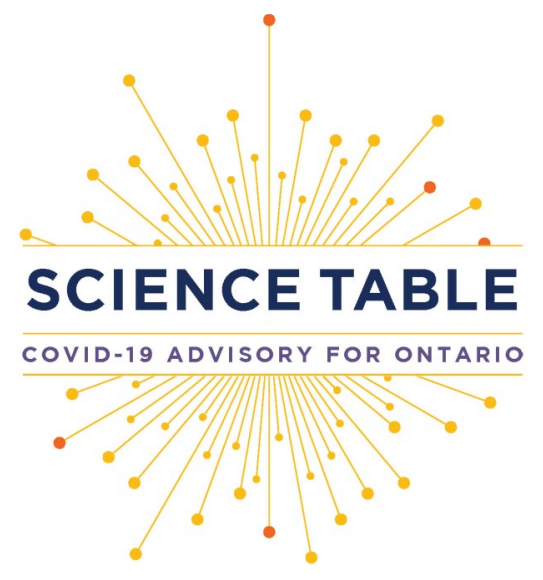

Version 1.0

Published: April 23, 2021

Updated on April 26, 2021.

Citation: Mishra S, Stall NM, Ma $\mathrm{H}$, et al. A vaccination strategy for Ontario COVID-19 hotspots and essential workers. Science Briefs of the Ontario COVID-19 Science Advisory Table. 2021;2(26). https:// doi.org/10.47326/ocsat.2021.02.26.1.0

Author Affiliations: The affiliations of the members of the Ontario COVID-19 Science Advisory Table can be found at https:// covid19-sciencetable.ca/.

Declarations of Interest: The declarations of interest of the members of the Ontario COVID-19 Science Advisory Table can be found at https://covid19-sciencetable.ca/. The declarations of interest of external authors can be found under additional resources at https:// doi.org/10.47326/ocsat.2021.02.26.1.0.

About Us: The Ontario COVID-19 Science Advisory Table is a group of scientific experts and health system leaders who evaluate and report on emerging evidence relevant to the COVID-19 pandemic, to inform Ontario's response. Our mandate is to provide weekly summaries of relevant scientific evidence for the COVID-19 Health Coordination Table of the Province of Ontario, integrating information from existing scientific tables, Ontario's universities and agencies, and the best global evidence. The Science Table summarizes its findings for the Health Coordination Table and the public in Science Briefs.

Correspondence to: Secretariat of the Ontario COVID-19 Science Advisory Table (info@covid19-sciencetable.ca)

Copyright: 2021 Ontario COVID-19 Science Advisory Table. This is an open access document distributed under the terms of the Creative Commons Attribution License, which permits unrestricted use, distribution, and reproduction in any medium, provided that the original work is properly cited.

The views and findings expressed in this Science Brief are those of the authors and do not necessarily reflect the views of all of the members of the Ontario COVID-19 Science

\title{
A Vaccination Strategy for Ontario COVID-19 Hotspots and Essential Workers
}

Sharmistha Mishra, Nathan M. Stall, Huiting Ma, Ayodele Odutayo, Jeffrey C. Kwong, Upton Allen, Kevin A. Brown, Isaac I. Bogoch, Aysegul Erman, Tai Huynh, Sophia Ikura, Antonina Maltsev, Allison McGeer, Gary Moloney, Andrew M. Morris, Michael Schull, Arjumand Siddiqi, Janet Smylie, Tania Watts, Kristy Yiu, Beate Sander, Peter Jüni on behalf of the Ontario COVID-19 Science Advisory Table

\section{Key Message}

Ontario's initial mass COVID-19 vaccination strategy in place until April 8, 2021 was based on per-capita regional allocation of vaccines with subsequent distribution - in order of relative priority - by age, chronic health conditions and high-risk congregate care settings, COVID-19 hotspots, and essential worker status.

Early analysis of Ontario's COVID-19 vaccine rollout reveals inequities in vaccine coverage across the province, with residents of higher risk neighbourhoods being least likely to get vaccinated.

Accelerating the vaccination of COVID-19 hotspots and essential workers will prevent considerably more SARS-CoV-2 infections and COVID-19 hospitalizations, ICU admissions and deaths as compared with Ontario's initial mass vaccination strategy (Figure 1).

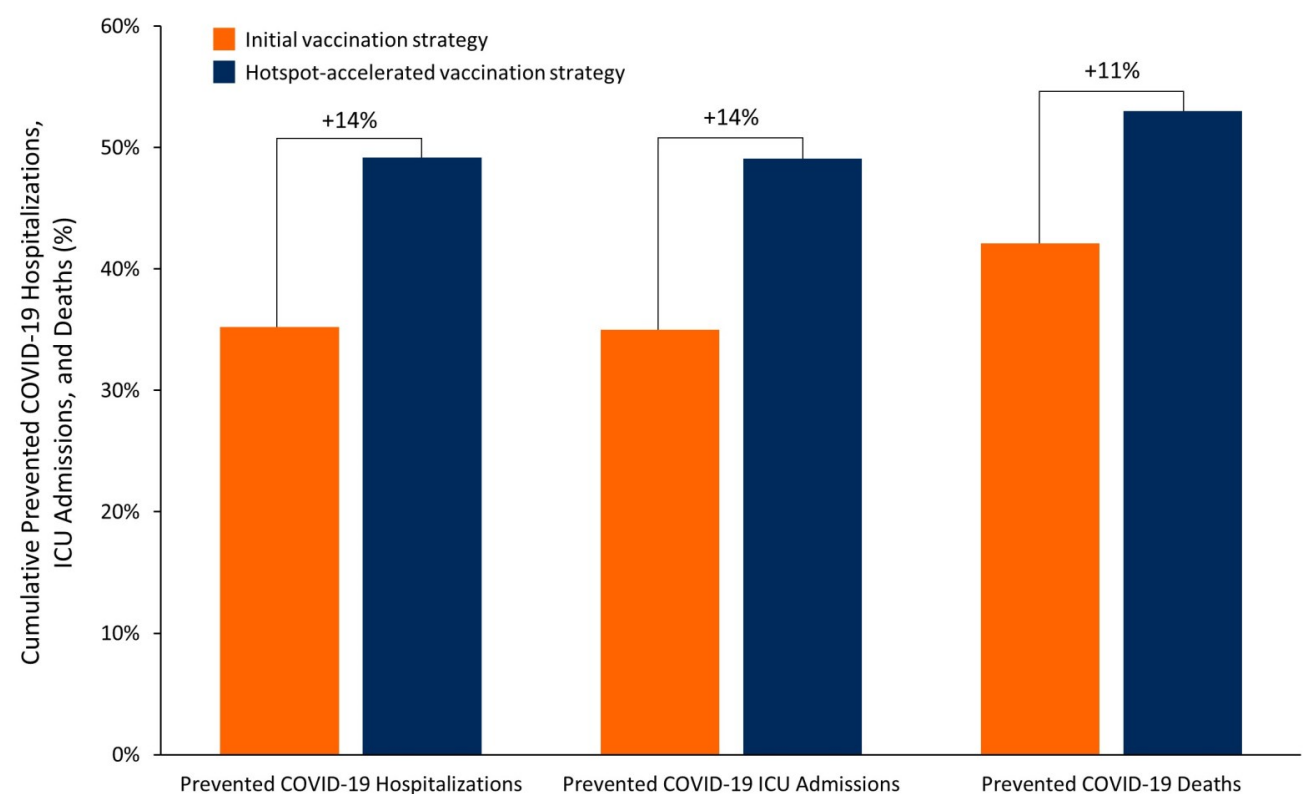

Figure 1. Percentage of COVID-19 Hospitalizations, ICU Admissions and Deaths Prevented by Vaccinating 3 Million Ontarians Comparing the Initial Vaccination Strategy with a Strategy Accelerating the Vaccination of Residents and Essential Workers in Hotspots

Bar chart showing the percentage of COVID-19 hospitalizations, ICU admissions and deaths that could be prevented by vaccinating 3 million Ontarians according to the initial mass vaccination strategy, which was in place until April 8 , 2021, and by accelerating vaccination of residents and essential workers in hotspots. We projected the cumulative number of COVID-19 hospitalizations, ICU admissions and deaths prevented at 60 days after the start of a 30-day vaccination period with the two strategies relative to a scenario of no further vaccination from April 9, 2021, onwards. The difference between strategies is expressed as an absolute difference in percentage of prevented event. 


\section{Summary}

\section{Background}

Ontario's initial mass COVID-19 vaccination strategy in place until April 8, 2021 was based on per-capita regional allocation of vaccines with subsequent distribution - in order of relative priority - by age, chronic health conditions and high-risk congregate care settings, COVID-19 hotspots, and essential workers in nonhealthcare settings. As of April 5, 2021, 15\% of Ontarians had received at least one dose of a COVID-19 vaccine.

Recent evidence indicates that COVID-19 vaccines not only protect directly against symptomatic COVID-19, but also against asymptomatic SARS-CoV-2 infection and against transmission to others. The indirect impact (benefits accrued even by those who are not yet vaccinated) can be captured by transmission models to estimate the direct and indirect impact of vaccination strategies.

\section{Questions}

How has COVID-19 vaccination coverage varied between neighbourhoods with different COVID-19 risks?

How have per-capita rates of SARS-CoV-2 cases differed between neighbourhoods with high and low proportions of essential workers in non-healthcare settings?

Could accelerating the vaccination of essential workers, their families and other residents living in COVID-19 hotspots prevent more SARS-CoV-2 infections and COVID-19 hospitalizations, ICU admissions and deaths as compared with Ontario's initial mass vaccination strategy?

\section{Findings}

By April 5, 2021, residents of neighbourhoods with the lowest risk of SARS-CoV-2 infections were 1.5 times more likely to have received at least one COVID-19 vaccine dose, as compared to residents of neighbourhoods with the highest risk of SARS-CoV-2 infections.

In Toronto, neighbourhoods with the highest proportion of essential workers in nonhealthcare settings continue to experience a 3-fold higher incidence of SARS-CoV-2 infections as compared to neighbourhoods with fewer essential workers.

Accelerating the vaccination of essential workers, their families and other residents living in COVID-19 hotspots is projected to prevent considerably more infections in those aged 16 to 59 years. Even though the hotspot-accelerated strategy results in slower vaccination of individuals aged 60 years and above in non-hotspots, it prevents more infections also in this age group as a result of faster vaccination in hotspots (direct impact), and prevention of infections passed from younger to older adults (indirect impact). A hotspot-accelerated vaccination strategy is also projected to prevent a larger number of COVID-19 hospitalizations, ICU admissions and deaths than Ontario's initial vaccination strategy.

\section{Interpretation}

Early analysis of Ontario's COVID-19 vaccine rollout reveals inequities in vaccine coverage across the province, with residents of neighbourhoods with higher COVID19 risk being least likely to get vaccinated. At the same time, COVID-19 continues to disproportionately impact neighbourhoods with the highest proportion of essential workers. 
Accelerating the vaccination of residents and essential workers in COVID-19 hotspots will substantially reduce the overall incidence of SARS-CoV-2 infections, COVID-19 hospitalizations, ICU admissions and deaths among both younger and older Ontarians.

\section{Background}

In Ontario, half of all SARS-CoV-2 infections are in $20 \%$ of neighbourhoods. ${ }^{1}$ These neighbourhoods are referred to as COVID-19 hotspots. COVID-19 hotspots have the highest incidence of infections, they are also the neighborhoods with the highest proportion of essential workers who are unable to work from home and must travel to their place of employment. ${ }^{2}$ Risk factors for heightened acquisition and transmission in these neighborhoods include lower-income, multigenerational households, high density housing, precarious working conditions, a lack of paid sick leave, and difficulty in access to or uptake of interventions such as isolation, quarantine and testing. ${ }^{3-5}$

Ontario's initial mass COVID-19 vaccination strategy in place until April 8, 2021 was based on per capita regional allocation of vaccines with subsequent distribution - in order of relative priority - by age, chronic health conditions and high-risk congregate care settings, COVID-19 hotspots, and essential workers in nonhealthcare settings.

As of April 5, 2021, 15\% of Ontarians have received at least one dose of a COVID-19 vaccine. Residents of COVID-19 hotspots and essential workers experience barriers to accessing COVID-19 vaccination. Essential workers cannot work from home, and are employed in the trades, transport, equipment, manufacturing, utilities, sales, services, agricultural, and health care sectors. ${ }^{6}$

Recent evidence indicates that COVID-19 vaccines not only protect against symptomatic COVID-19, ${ }^{7,8}$ but also against asymptomatic SARS-CoV-2 infection and thus, transmission..$^{9-11}$ Accelerated vaccination of a large proportion of residents and essential workers in COVID-19 hotspots has the potential to not only result in direct benefits for vaccinated individuals (prevention of symptomatic disease), but also in indirect benefits for unvaccinated individuals (prevention of transmission from vaccinated to unvaccinated individuals). These indirect benefits can now be incorporated into models to estimate the impact of different vaccination strategies. As an example, transmission modeling from British Columbia and Spain suggests considerable population-level benefit for all age-groups of vaccinating those who may be most at risk of acquisition and transmission of SARS-CoV-2, such as essential workers. ${ }^{12,13}$

\section{Questions}

How has COVID-19 vaccination coverage varied between neighbourhoods with different COVID-19 risks?

How have per-capita rates of SARS-CoV-2 cases differed between neighbourhoods with high and low proportions of essential workers in non-healthcare settings?

Could accelerating the vaccination of essential workers, their families and other residents living in COVID-19 hotspots prevent more SARS-CoV-2 infections and COVID-19 hospitalizations, ICU admissions and deaths as compared with Ontario's initial mass vaccination strategy?

\section{Findings}

Across Ontario, neighbourhoods with the highest risk of SARS-CoV-2 infection had the lowest rates of COVID-19 vaccination. Figure 2 presents the COVID-19 vaccination 
coverage of Ontarians aged 16 years and above by neighborhood COVID-19 risk. Residents of neighbourhoods with the lowest risk of SARS-CoV-2 infections (COVID-19 neighbourhood risk group 10) were 1.5 times more likely to have received at least one COVID-19 vaccine dose by April 5, 2021, as compared to residents of neighbourhoods with the highest risk of SARS-CoV-2 infections (COVID-19 neighbourhood risk group 1, Figure 2A). ${ }^{1}$ However, the average incidence of SARS-CoV-2 infections between March 7 and April 3, 2021 was 9 times lower in neighbourhoods with the lowest risk of SARSCoV-2 infections (Figure 2B).
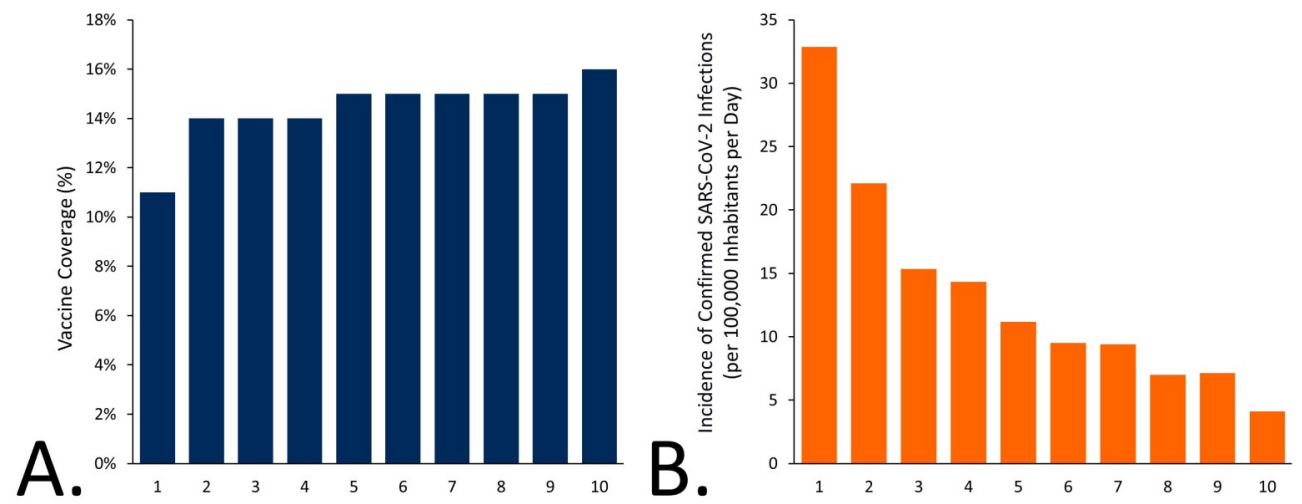

COVID-19 Neighbourhood Risk Groups (1 = highest incidence, 10- lowest incidence)

Figure 2. COVID-19 Vaccine Coverage and Incidence of Confirmed SARS-CoV-2 Infections in Ontario as of early April 2021 Panel A presents COVID-19 vaccine coverage in Ontario for inhabitants aged 16 years and older, as of April 5, 2021, by neighbourhood COVID-19 risk group. Panel B presents the average incidence of confirmed SARS-CoV-2 infections per 100,000 inhabitants in Ontario per day, from March 7 to April 3, 2021, by neighbourhood COVID-19 risk group. Neighbourhood risk groups are determined by ranking Ontario's 509 neighbourhoods in 10\% increments according to the cumulative incidence of SARS-CoV-2 infections between Jan 23, 2020 and Jan 16, 2021. ${ }^{1}$ Each risk group represents $10 \%$ of the Ontario population ( 1.5 million inhabitants). Group 1 includes neighbourhoods with the highest cumulative incidence of SARS-CoV-2 infection, whereas group 10 includes neighbourhoods with the lowest cumulative incidence of SARS-CoV-2 infection. Data sourced from the Public Health Case and Contact Management Solution and other case management systems (CCM plus) and the ICES COVID-19 Dashboard. ${ }^{14}$

Figure 3 presents the incidence of new SARS-CoV-2 infections based on the proportion of essential workers in non-healthcare settings living in Toronto's neighbourhoods, separated into thirds based on the proportion of essential workers in non-healthcare settings. ${ }^{2}$ Neighbourhoods with the highest proportion of essential workers in non-healthcare settings continued to experience a 3-fold higher incidence of SARS-CoV-2 infections as compared to Toronto neighbourhoods with the lowest proportion of essential workers. These neighbourhoods also have the highest proportion of multi-generational households.

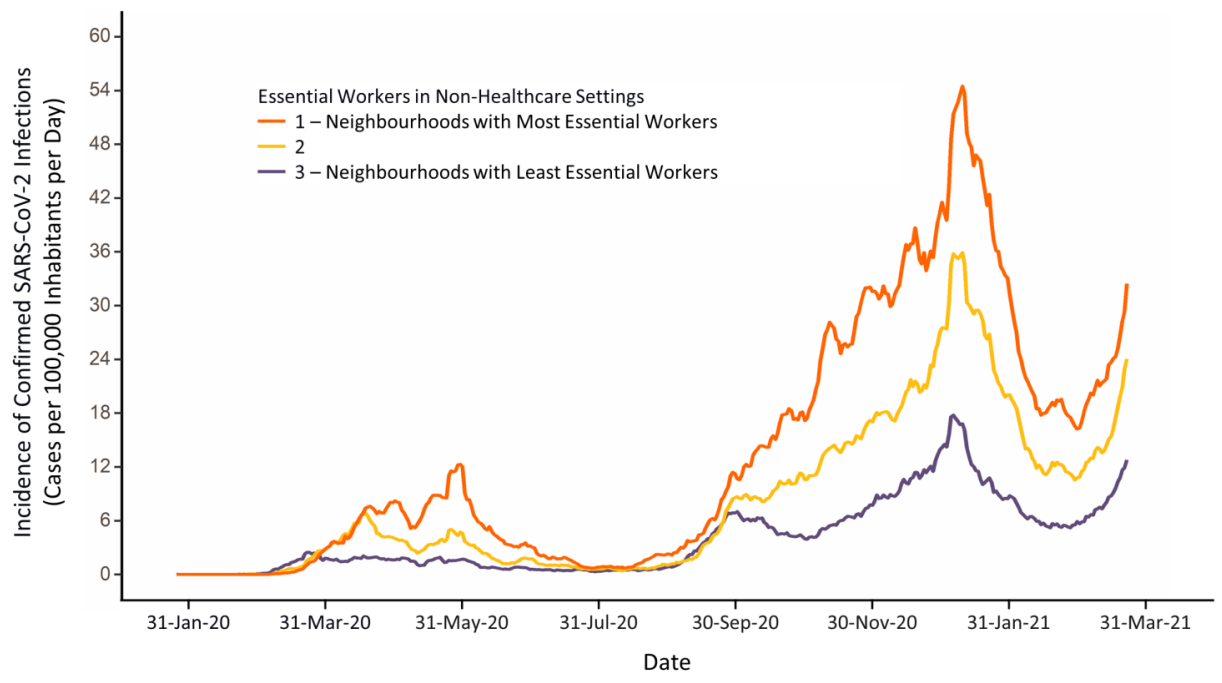

Figure 3. Daily Incidence of Confirmed SARS-CoV-2 Infections by Proportion of Essential Workers in NonHealthcare Settings Living in Toronto's Neighbourhoods, January 20, 2020 to March 23, 2021 
Line graph presenting the 7-day moving average of confirmed SARS-CoV-2 infections per 100,000 inhabitants in the City of Toronto from January 20, 2020 to March 23, 2021 excluding long-term care (LTC) home residents. Neighbourhoods are separated into thirds, based on the proportion of essential workers in non-healthcare settings living in the neighbourhoods. The top third represents the 33\% of neighbourhoods (by overall population size) with the highest proportion of essential non-healthcare workers; the bottom third represents the 33\% of neighbourhoods (by overall population size) with the lowest proportion of essential non-healthcare workers. Neighbourhoods are defined by dissemination areas of 400 to 700 residents. ${ }^{4,15}$ Data sourced from Case and Contact Management Solutions (CCM)+ and the Statistics Canada 2016 Census.

Figure 4 compares the projected impact of two vaccination scenarios: Ontario's initial COVID-19 vaccination strategy versus a strategy that accelerates the vaccination of essential workers, their families and other residents living in COVID19 hotspots. In both scenarios, 3 million doses of COVID-19 vaccine are administered over 30 days $(100,000$ doses per day starting on April 9, 2021), and the number of new SARS-CoV-2 infections over 60 days (April 9 to June 7, 2021) is determined.

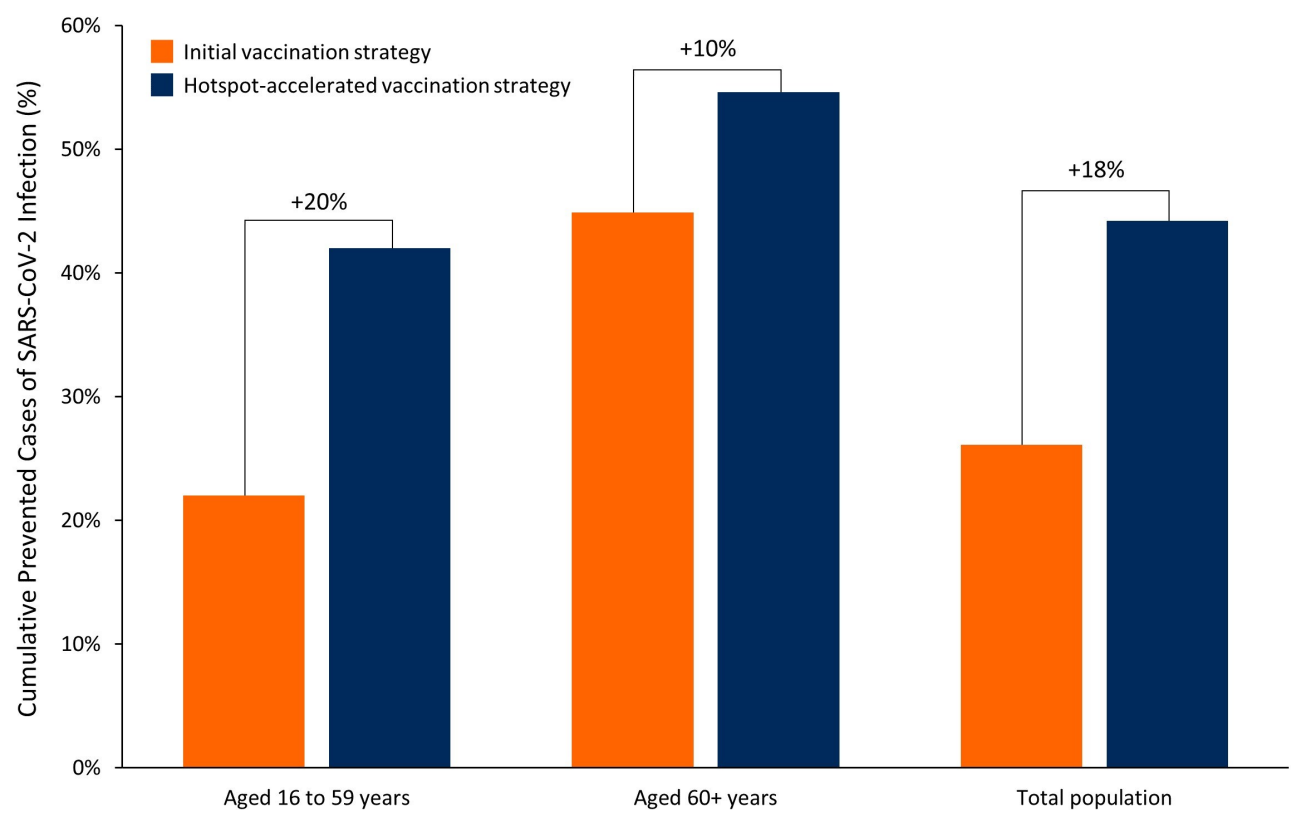

Figure 4. Percentage of SARS-CoV-2 Infections Prevented by Vaccinating 3 Million Ontarians Comparing the Initial Vaccination Strategy with a Strategy Accelerating the Vaccination of Residents and Essential Workers in Hotspots Bar chart showing the percentage of SARS-CoV-2 infections prevented by vaccinating 3 million Ontarians according to the initial vaccination strategy ${ }^{16,17}$ and according to a strategy accelerating the vaccination of essential workers, their families and other residents living in COVID-19 hotspots. We projected the number of SARS-CoV-2 infections prevented with the two vaccination strategies relative to a scenario of no further vaccination from April 9, 2021, onwards. The difference between strategies is expressed as an absolute difference in percentage of prevented event.

Ontario's initial mass COVID-19 vaccination strategy emphasized age-based prioritization, with vaccines distributed equally across the ten COVID-19 neighbourhood risk groups, consistent with the province's per capita allocation of vaccines. ${ }^{18}$ For the initial strategy, we assumed that $80 \%$ of the population aged 60 years and above would be vaccinated by April 28, 2021, at which point the strategy would proceed to vaccinating individuals aged 16 to 59 years of age. In the initial strategy, ${ }^{16,17}$ an additional 900,000 doses were allocated to COVID-19 hotspots. We assumed that these allocated doses would be distributed at a rate of 450,000 doses per month, and would be administered to produce equal coverage across the neighbourhood risk groups, mitigating the previously observed disparities in vaccine coverage across the groups (see Figure 2).

In the hotspot-accelerated vaccination strategy, we assumed that 50\% of COVID-19 vaccines $(50,000$ per day) would be distributed to the 74 COVID-19 hotspots in neighbourhood risk groups 1 and 2 (the 20\% of neighbourhoods with the highest cumulative incidence of SARS-CoV-2). ${ }^{1}$ We further assumed that these vaccines would be distributed to individuals aged 16 years and above with no age-based 
prioritization in these hotspots. For the remaining 50\% of COVID-19 vaccines we assumed equal distribution across all ten COVID-19 neighbourhood risk groups, and that these vaccines would be used to vaccinate individuals aged 60 years and above in all neighbourhoods, including hotspots, with an age-based prioritization approach. With this strategy, the vaccination of the 74 hotspots would be completed within 25 days, assuming vaccine coverage of $80 \%$ in those aged 60 years and above, and $70 \%$ in those aged 16 to 59 years. All vaccine doses available after 25 days would then be distributed equally across the remaining neighbourhoods.

Figure 4 indicates that compared to Ontario's initial COVID-19 vaccination strategy, a strategy that accelerates vaccination of residents and essential workers in COVID19 hotspots would prevent a larger number of SARS-CoV-2 infections between April 9 and June 7,2021 . The hotspot-accelerated vaccination strategy is also projected to prevent a larger number of infections in individuals aged 16 to 59 years and among individuals aged 60 years and above even though the strategy results in slower vaccination of individuals aged 60 years and above.

\begin{tabular}{lccc}
\hline & $\begin{array}{c}\text { No vaccination beyond } \\
\text { April 8, 2021 }\end{array}$ & $\begin{array}{c}\text { Continue Ontario's initial } \\
\text { COVID-19 vaccination } \\
\text { strategy }\end{array}$ & $\begin{array}{c}\text { Hotspot-accelerated } \\
\text { vaccination strategy }\end{array}$ \\
\hline SARS-CoV-2 infections & 313,124 & 231,531 & 174,738 \\
COVID-19 hospitalizations & 12,352 & 8,003 & 6,272 \\
COVID-19 ICU admissions & 1,833 & 1,191 & 933 \\
COVID-19 deaths & 4,740 & 2,745 & 2,228 \\
\hline
\end{tabular}

Table 1. Cumulative Projected SARS-CoV-2 Cases, COVID-19 Hospitalizations, ICU Admissions and Deaths in Ontario Cumulative projected SARS-CoV-2 cases, COVID-19 hospitalizations, ICU admissions and deaths in Ontario from April 9 to June 7, 2021, under three scenarios: 1) no additional vaccination beyond April 8, 2021, 2) continue Ontario's initial COVID-19 vaccination strategy, and 3) a hotspot-accelerated strategy that accelerates the vaccination of essential workers in non-healthcare settings, their families and other residents living in COVID-19 hotspots.

Figure 1 and Table 1 present the projected impact of the two vaccination strategies compared with no vaccinations beyond April 8, 2021. Projected outcomes include the number of COVID-19 hospitalizations, ICU admissions, and deaths in Ontario between April 9 and June 7, 2021. Compared with the initial strategy, the hotspotaccelerated vaccination strategy is projected to prevent an additional $14 \%$ of COVID19 hospitalizations, $14 \%$ of COVID-19 ICU admissions and 11\% of COVID-19 deaths in Ontario's population aged 16 years and older. With 3 million doses available over 30 days, the number of vaccinations needed to prevent 1 confirmed SARS-CoV-2 infection is 37 with the initial strategy and 22 with the hotspot-accelerated strategy.

\section{Interpretation}

Early analysis of Ontario's COVID-19 vaccine rollout reveals inequities in vaccine coverage across the province. The impact of COVID-19 is highest in Ontario neighbourhoods with the largest proportion of essential workers. ${ }^{3}$ As of April 5, 2021, residents of neighbourhoods with the highest risk of SARS-CoV-2 infection had the lowest rates of COVID-19 vaccination.

Recent evidence indicates that COVID-19 vaccines not only protect against symptomatic COVID-19, 7,8 but also against asymptomatic SARS-CoV-2 infection and transmission. $^{9-11}$ These indirect benefits were incorporated into models that compare vaccination strategies that leverage both the direct and indirect benefits of vaccination.

Accelerating the vaccination of essential workers, their families and other residents living in COVID-19 hotspots by immediately reallocating $50 \%$ of the next 3 million vaccines to the $20 \%$ of neighbourhoods with the highest cumulative incidence of SARS-CoV-2, and vaccinating all residents aged 16 years and above in these 
neighbourhoods could substantially reduce the overall incidence of SARS-CoV-2 infections, COVID-19 hospitalizations, ICU admissions and deaths in both younger and older Ontarians. This is because the hotspot-accelerated vaccination strategy not only affords more direct protection to Ontario's younger residents and essential workers in COVID-19 hotspots, but it also affords substantially more indirect protection to older adults in these hotspots. ${ }^{12}$ Essential workers experience a disproportionately high burden of SARS-CoV-2 infection, especially in the absence of paid sick leave and enhanced supports for workplace safety. ${ }^{2}$ Older adults, especially those living in multigenerational households, will benefit from fewer infections among younger adults, many of whom are essential workers.

On April 13, 2021, Ontario communicated an updated COVID-19 mass vaccination strategy to accelerate the vaccination of COVID-19 hotspots. The updated strategy involves vaccinating adults aged 18 years and older in the highest risk hotspots identified in Toronto and Peel, and later vaccinating the remainder of 114 defined hotspots across the province. ${ }^{19}$ Of note, the province defined hotspots predominantly based on the top three COVID-19 neighbourhood risk groups identified in the Science Brief entitled A Strategy for the Mass Distribution of COVID19 Vaccines in Ontario Based on Age and Neighbourhood, ${ }^{1}$ with neighbourhoods ranked in the top $30 \%$ of cumulative SARS-CoV-2 incidence. In addition, they based the selection of hotspots on outbreak data and a list of neighbourhoods with low testing rates and sociodemographic barriers that may result in lower vaccine uptake. ${ }^{20,21}$ Ontario's updated vaccination strategy would distribute $25 \%$ of vaccines to the identified 114 hotspots and the remaining $75 \%$ would be equally distributed across the province (including hotspots). ${ }^{22}$ This means that $47.5 \%$ of Ontario's COVID -19 vaccine supply would be allocated to the $30 \%$ of Ontario's population living in 114 hotspots identified by the province.

The hotspot-accelerated vaccination strategy suggested in this Science Brief would allocate $50 \%$ of COVID-19 vaccines to the 74 COVID-19 hotspots ranked in the top $20 \%$ of cumulative SARS-CoV-2 incidence among all Ontario neighbourhoods, and the remaining $50 \%$ of vaccines would be equally distributed across the province (including hotspots). ${ }^{1}$ This means that $60 \%$ of Ontario's COVID-19 vaccine supply would be allocated to the $20 \%$ of Ontario's population living in the hotspots identified by the Ontario COVID-19 Science Advisory Table. ${ }^{1}$ Additional benefits would be realized by further prioritizing warehouses, factories and other facilities with large numbers of COVID-19 outbreaks.

There are important caveats to the results of our analyses. The highest COVID-19 risk neighbourhoods were modeled separately from remaining neighbourhoods. As such, our estimates underestimate the potential benefit of the hotspot-accelerated strategy on the overall population: we do not explicitly capture the indirect benefits of the prevention of infection spillover from hotspots into low-risk neighbourhoods. Our model also does not explicitly stratify the adult population into individuals who are essential workers and those who are not. However, the model accounts for higher rates of workplace-related contacts since COVID-19 hotspots have a higher proportion of essential workers.

Realizing the potential impact of the hotspot-accelerated vaccination strategy will require meaningful community engagement, investment in, and support for, the implementation and logistics of community-level vaccine distribution. Examples of community-level distribution include implementation through trusted and established community programs, health-care providers (especially primary care physicians, nurse practitioners, and community health centers), and employer-based programs for essential workers. 


\section{Methods Used for This Science Brief}

We developed an age-stratified mathematical model of SARS-CoV-2 transmission, using a modified SEIR system of compartments (susceptible, exposed, infectious, and recovered) with COVID-19 vaccination. The model simulated contacts within and between two age-groups (age 16-59 years of age; and 60 years of age and above) and separately modelled high SARS-CoV-2 incidence neighbourhoods or "COVID-19 hotspots" (20\% of the Ontario population over age 16) and lowerincidence neighbourhoods ( $80 \%$ of the Ontario population over age 16).

The model used Ontario demographic and surveillance data on the hotspots (two highest SARS-CoV-2 incidence neighbourhood groups as per Brown et al. ${ }^{1}$ ) and the remaining forward sortation areas (FSAs, eight lowest SARS-CoV-2 incidence neighbourhood groups as per Brown et al. ${ }^{1}$ ), to reflect the following summary measures: estimated reproductive rates and relative rates of cumulative and weekly SARS-CoV-2 infections (in March 2021) between COVID-19 hotspots and lowerincidence FSAs; cumulative proportion of total provincial cases among over age 16 years that are in the hotspots (50-55\%); relative rates of cumulative and weekly diagnoses (in March 2021) between age-groups within and between hotspots and lower-incidence FSAs. ${ }^{1}$

Inputs on contact rates and patterns by age-group were based on the CONNECT study but calibrated to the effective reproduction number in the hotspots and lower SARS-CoV-2 incidence neighbourhoods. ${ }^{23}$ As such, rates of contacts were higher in the COVID-19 hotspots stemming from a larger proportion of the population who are not able to work remotely (essential workers).

Vaccination was assumed to reduce acquisition of infection with an efficacy of $60 \%$ after one dose..$^{9-11}$ The model captured direct and indirect benefits of vaccinations within the hotspots, and within the lower SARS-CoV-2 incidence FSAs. The model included the current proportion of the age-stratified population in COVID-19 hotspots and lower SARS-CoV-2 incidence neighbourhoods estimated as immune following infection ("recovered"), current proportion infectious (active SARS-CoV-2 cases diagnosed and undiagnosed), and current proportion who have received at least one dose of a COVID-19 vaccine as of April 5, 2021 based on the ICES Dashboard. ${ }^{14}$ With this approach the model will underestimate the impact of COVID19 vaccination in one region (COVID-19 hotspots) on lower-SARS-CoV-2 incidence neighbourhoods, and vice-versa; as the previous model assumes independence of COVID-19 hotspots and lower SARS-CoV-2 incidence neighbourhoods. Vaccination was assumed to reduce acquisition of infection with an efficacy of $60 \% .{ }^{9-11}$ The model captured direct and indirect benefits of vaccinations within the hotspots, and within the lower-incidence FSAs. The model included the current proportion of the age-stratified population in COVID-19 hotspots and lower SARS-CoV-2 incidence neighbourhoods estimated as immune following infection ("recovered"), current proportion infectious (active SARS-CoV-2 cases diagnosed and undiagnosed), and current proportion who have received at least one dose of a COVID-19 vaccine as of April 5, 2021 based on the ICES Dashboard. ${ }^{14}$ With this approach the model will underestimate the impact of COVID-19 vaccination in one region (COVID-19 hotspots) on lower-SARS-CoV-2 incidence neighbourhoods, and vice-versa; as the previous model assumes independence of COVID-19 hotspots and lower SARS-CoV-2 incidence neighbourhoods.

The model was then used to simulate counterfactual scenarios wherein the basecase (no further COVID-19 vaccination beyond April 8, 2021) was compared with a fixed amount of daily vaccinations $(100,000$ per day) distributed using the initial 
strategy. For this strategy, we assumed that $80 \%$ of the population aged 60 years and above would be vaccinated by April 28,2021 , at which point the strategy would proceed to vaccinating individuals aged 16 to 59 years of age. In the initial strategy, ${ }^{16,17}$ an additional 900,000 doses were allocated to COVID-19 hotspots. We assumed that these allocated doses would be distributed at a rate of 450,000 doses per month, and would be administered to produce equal coverage across the neighbourhood risk groups, mitigating the previously observed disparities in vaccine coverage across the groups (see Figure 2).

The hotspot-accelerated vaccination strategy was assumed to allocate $50 \%$ of COVID -19 vaccines to the 74 COVID-19 hotspots ranked in the top $20 \%$ of cumulative SARSCoV-2 incidence among all Ontario neighbourhoods, and the remaining $50 \%$ of vaccines would be equally distributed across the province (including hotspots). ${ }^{1}$ This means that $60 \%$ of Ontario's COVID-19 vaccine supply would be allocated to the $20 \%$ of Ontario's population living in the hotspots identified by the Ontario COVID19 Science Advisory Table. ${ }^{1}$

We then compared the difference in infections averted, and applied age-specific COVID-19 hospitalization, need for ICU, and mortality based on Ontario data and under the assumption that $>80 \%$ of cases would be a variant of concern (specifically B.1.1.7). ${ }^{1,24,25}$. We assumed that age-specific metrics for COVID-19 severity (hospitalization, ICU, and mortality) were the same in COVID-19 hotspots and lower SARS-CoV-2 incidence neighbourhoods although data suggest age-specific casefatality rates remain higher in lower-income neighbourhoods even after adjustment for comorbidities. ${ }^{10}$ Thus, impact may reflect an underestimate of severity prevented within COVID-19 hotpots.

\section{Author Contributions}

SM, BS and PJ conceived the Science Brief. SM, NMS, AO and PJ wrote the first draft of the Science Brief. SM, KAB, BS, HM, GM, AE, and PJ performed the analyses. All authors revised the Science Brief critically for important intellectual content and approved the final version. BS and PJ contributed equally.

\section{References}

1. Brown KA, Stall NM, Joh E, et al. A strategy for the mass distribution of COVID-19 vaccines in Ontario based on age and neighbourhood. Sci Briefs Ont COVID-19 Sci Advis Table. 2021;2(10). https://doi.org/10.47326/ocsat.2021.02.10.1.0

2. Chagla Z, Ma H, Sander B, Baral SD, Mishra S. Characterizing the disproportionate burden of SARS-CoV-2 variants of concern among essential workers in the Greater Toronto Area, Canada. medRxiv. Published online March 26, 2021:2021.03.22.21254127. https://doi.org/10.1101/2021.03.22.21254127

3. Sundaram $M E$, Calzavara $A$, Mishra $S$, et al. The individual and social determinants of COVID-19 in Ontario, Canada: a population-wide study. medRxiv. Published online November 12, 2020:2020.11.09.20223792. https:// doi.org/10.1101/2020.11.09.20223792

4. Mishra S, Ma H, Moloney G, et al. Increasing concentration of COVID-19 by socioeconomic determinants and geography in Toronto, Canada: an observational study. medRxiv. Published online April 6, 2021:2021.04.01.21254585. https://doi.org/10.1101/2021.04.01.21254585

5. Paul LA, Daneman N, Brown KA, et al. Characteristics associated with household transmission of SARS-CoV-2 in Ontario, Canada. medRxiv. Published online 
October 26, 2020:2020.10.22.20217802. https://

doi.org/10.1101/2020.10.22.20217802

6. Government of Canada. Occupational classifications. Statistics Canada. Published November 21, 2011. https://www.statcan.gc.ca/eng/concepts/ occupation

7. Baden LR, El Sahly HM, Essink B, et al. Efficacy and safety of the mRNA-1273 SARS-CoV-2 vaccine. N Engl J Med. Published online December 30, 2020. https:// doi.org/10.1056/NEJMoa2035389

8. Polack FP, Thomas SJ, Kitchin N, et al. Safety and efficacy of the BNT162b2 mRNA COVID-19 vaccine. N Engl J Med. Published online December 10, 2020. https://doi.org/10.1056/NEJMoa2034577

9. Hall VJ, Foulkes S, Saei A, et al. Effectiveness of BNT162b2 mRNA vaccine against Infection and COVID-19 vaccine voverage in healthcare workers in England, multicentre prospective cohort study (the SIREN study). SSRN. Published online February 22, 2021. https://doi.org/10.2139/ssrn.3790399

10. Thompson MG. Interim Estimates of Vaccine Effectiveness of BNT162b2 and mRNA-1273 COVID-19 Vaccines in Preventing SARS-CoV-2 Infection Among Health Care Personnel, First Responders, and Other Essential and Frontline Workers - Eight U.S. Locations, December 2020-March 2021. MMWR Morb Mortal Wkly Rep. 2021;70. https://doi.org/10.15585/mmwr.mm7013e3

11. Dagan N, Barda N, Kepten E, et al. BNT162b2 mRNA Covid-19 Vaccine in a Nationwide Mass Vaccination Setting. N Engl J Med. Published online February 24, 2021. https://doi.org/10.1056/NEJMoa2101765

12. Mulberry N, Tupper P, Kirwin E, McCabe C, Colijn C. Vaccine Rollout Strategies: The Case for Vaccinating Essential Workers Early.; 2021. https://github.com/ nmulberry/essential-workers-vaccine/blob/main/vaccine_rollout_preprint.pdf

13. Rodríguez J, Patón M, Acuña JM. COVID-19 vaccination rate and protection attitudes can determine the best prioritisation strategy to reduce fatalities. medRxiv. Published online February 10, 2021:2020.10.12.20211094. https:// doi.org/10.1101/2020.10.12.20211094

14. ICES. COVID-19 Dashboard. Published 2021. Accessed July 24, 2020. https:// www.ices.on.ca/DAS/AHRQ/COVID-19-Dashboard

15. Rao A, Ma H, Moloney G, et al. A disproportionate epidemic: COVID-19 cases and deaths among essential workers in Toronto, Canada. medRxiv. Published online February 18, 2021:2021.02.15.21251572. https:// doi.org/10.1101/2021.02.15.21251572

16. McGrath JM. Here's what Ontario's latest vaccine plan says. TVO.org. Published March 5, 2021. https://www.tvo.org/article/heres-what-ontarios-latest-vaccineplan-says

17. Government of Ontario. Ontario moving to Phase Two of COVID-19 vaccine distribution plan. Published April 6, 2021. https://news.ontario.ca/en/ release/61009/ontario-moving-to-phase-two-of-covid-19-vaccine-distributionplan

18. Government of Ontario. COVID-19: Guidance for Prioritization of Phase 2 Populations for COVID-19 Vaccination. Ministry of Health; 2021. Accessed April 7, 2021. https://www.health.gov.on.ca/en/pro/programs/publichealth/ coronavirus/docs/vaccine/COVID-19_Phase_2_vaccination_prioritization.pdf 
19. McGrath JM. Here's Ontario's revised Phase 2 vaccination plan. TVO.org. Published April 13, 2021. https://www.tvo.org/article/heres-ontarios-revisedphase-2-vaccination-plan

20. Crawley M, April 132021 6:09 AM ET | Last Updated: These "hot spots" getting vaccine priority are less hard-hit by COVID-19 than Ontario average. CBC. Published April 12, 2021. https://www.cbc.ca/news/canada/toronto/ontariocovid-19-vaccination-postal-code-hot-spots-1.5983155

21. Warren M, Wallace K, Tulk C. Ontario ignored its own Science Table's advice on several COVID-19 vaccine hotspot postal codes. thestar.com. Published April 20, 2021. https://www.thestar.com/news/gta/2021/04/20/ontario-ignored-its-own -science-tables-advice-on-several-covid-19-vaccine-hotspot-postal-codes.html

22. Global News. Ontario increasing COVID-19 vaccine supply to 'hot spots,' Ford says. Global News. Published April 16, 2021. https://globalnews.ca/ video/7764570/ontario-increasing-covid-19-vaccine-supply-to-hot-spots-fordsays/

23. Research Group in Mathematical Modeling and Health Economics of Infectious Disease. COVID-19 pandemic. Published 2021. https://marc-brisson.net/

24. Tuite AR, Fisman DN, Odutayo A, et al. COVID-19 hospitalizations, ICU admissions and deaths associated with the new variants of concern. Sci Briefs Ont COVID-19 Sci Advis Table. 2021;1(18). https://doi.org/10.47326/ ocsat.2021.02.18.1.0

25. Mac S, Barrett K, Khan YA, et al. COVID-19 demographics, acute care resource use and mortality by age and sex in Ontario, Canada: population-based retrospective cohort analysis. medRxiv. Published online November 6, 2020:2020.11.04.20225474. https://doi.org/10.1101/2020.11.04.20225474

26. Kwong J, Calzavara A, Sundaram M, Mishra S. Predictors of Case Fatality among Individuals Who Have Tested Positive for COVID-19.; 2021:1. https://wellnesshub.ca/wp-content/uploads/Predictors-of-Case-Fatality-COVID-19-ResearchBrief-2.pdf 